\title{
Vascular Ehlers-Danlos Syndrome Presenting as a Pulsatile Neck Mass: a Case Report and Review of Literature
}

\author{
Bharat Maraj, MD, Emily Harding-Theobald, MD, and Fatima Karaki, MD \\ Department of Medicine, University of California, San Francisco Medical Center, San Francisco, CA, USA.
}

\begin{abstract}
Ehlers-Danlos syndrome refers to a spectrum of connective tissue disorders typically caused by mutations in genes responsible for the synthesis of collagen. Patients with Ehlers-Danlos syndrome often exhibit hyperflexibility of joints, increased skin elasticity, and tissue fragility. Vascular Ehlers-Danlos (vEDS) is a subtype of Ehlers-Danlos syndrome with a predilection to involve blood vessels. As such, it often manifests as vascular aneurysms and vessel rupture leading to hemorrhage. There are few reports describing primary prevention of aneurysms in the setting of undiagnosed, suspected vEDS. We present a case of a 30-year-old woman who presents with a pulsatile neck mass found to have multiple arterial aneurysms on imaging, hyperflexibility, and characteristic facial features consistent with vEDS. As described in this case, management of a suspected connective tissue disorder is a multidisciplinary approach including vascular surgery, medical therapy, and genetic testing to confirm the diagnosis. We review literature regarding the care of patients with vascular Ehlers-Danlos as it might pertain to hospitalized patients.
\end{abstract}

KEY WORDS: vascular; Ehlers-danlos; aneurysm.

$\mathrm{J}$ Gen Intern Med

DOI: $10.1007 / \mathrm{s} 11606-018-4445-3$

(C) Society of General Internal Medicine 2018, corrected publication August/2018

\section{INTRODUCTION}

Ehlers-Danlos syndrome is a heterogeneous group of rare connective tissue disorders that often manifest with increased skin elasticity, joint hypermobility, and tissue fragility. Vascular Ehlers-Danlos syndrome (vEDS) accounts for fewer than 5\% of all Ehlers-Danlos cases ${ }^{1}$. It is associated with an autosomal dominant mutation in COL3A1, responsible for the synthesis of type III collagen ${ }^{2-4}$. Type III collagen composes connective tissue found within all organs. It provides tensile strength for organs that must resist permanent dilation from fluctuations in pressure. Consequently, vEDS typically presents as rapidly progressive arterial aneurysms, arterial rupture, bowel perforation, and uterine fragility ${ }^{5-7}$. Suspicion often arises when incidental findings are identified on routine imaging studies ${ }^{6}$. Definitive diagnosis requires identification of a heterozygous

Received August 5, 2017

Revised December 8, 2017

Accepted April 10, 2018
COL3A1 pathogenic genotype. We report the case of a young woman who was diagnosed with a rapidly enlarging arterial pseudoaneurysm, ultimately found to have vEDS, managed with open ligation of her external carotid artery.

\section{CASE PRESENTATION}

A 30-year-old woman with a history of bilateral varicose veins was admitted from otolaryngology clinic with a 3-week history of a painful, rapidly growing right neck mass. The patient was previously diagnosed with parotitis at an outside hospital but the neck mass continued to grow despite completion of a two-week course of cephalexin. She denied trauma or injury to her neck, jaw, or face. She denied fevers, chills, weight loss, visual changes, and arthralgias. The patient had a medical history of persistent bilateral varicose veins despite uncomplicated endovenous ablation 1 year prior to admission. She also had a remote history of colonic perforation secondary to a lowspeed motor vehicle accident which required laparotomy and repair. She did note a history of easy bruising throughout her life. She had no family history of bleeding diathesis or connective tissue disease. On admission, her vitals were within normal limits. Her physical exam was significant for a $3-\mathrm{cm}$ diameter pulsatile mass with surrounding edema of the right neck and mandible. There was a violaceous, reticular rash overlying the mass (Fig. 1a). Skin elasticity was normal. Her face had large eyes, a thin nose, thin lips and a small chin. She had bilateral severe varicose veins without lower extremity edema (Fig. 1b). Distal interphalangeal joints and shoulders were hyperextensible with multiple movements (Fig. 1d, e). Metacarpophalangeal joints hyperextended $>90^{\circ}$, hyperflexion of wrists approached $140^{\circ}$, and her knees achieved hyperextension $>15^{\circ}$. Cardiovascular, pulmonary, abdominal, and neurologic examinations were unremarkable. Complete blood count, electrolytes, serum creatinine, liver function tests, and international normalized ratio were within normal limits. Blood cultures were negative. Erythrocyte sedimentation rate and C-reactive protein were not elevated. Antinuclear antibody, rheumatoid factor, anti-neutrophil cytoplasmic antibody, and complement levels were unremarkable.

Computer tomography angiography (CTA) of the neck revealed a $2.7-\mathrm{cm}$ aneurysm arising from the proximal right internal maxillary artery (Fig. 2) as well as a 4-mm aneurysm in the proximal right internal carotid artery. Brain CTA 

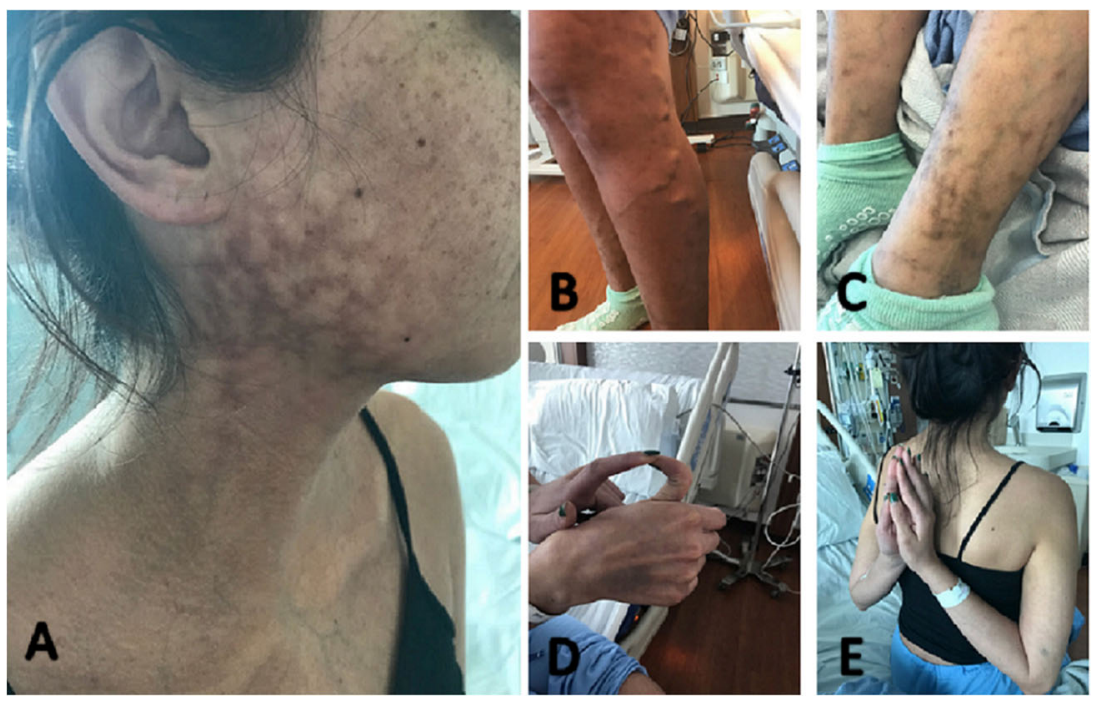

Figure 1. a Neck mass with overlying reticular rash. b Prominent varicose veins. c Easily identified veins suggestive of thin skin. d Interphalangeal joint hypermobility of the 1st digit. e Increased range of motion of shoulders.

showed a 3-mm aneurysm of the left middle cerebral artery. Chest, abdomen, and pelvis CTA did not reveal any other aneurysms. Transthoracic echocardiography did not show mitral valve prolapse or other abnormalities.

Rheumatology, otolaryngology, interventional radiology, and vascular surgery were consulted. The patient's clinical picture was thought to be most consistent with vEDS. After multidisciplinary discussions, the patient underwent an urgent open ligation of the maxillary and temporal artery via vascular surgery. The surgery was uncomplicated and the patient made a full recovery. Genetic testing was sent to confirm the diagnosis of vEDS. She was started on carvedilol, oral contraception, and was discharged with genetics follow-up and a CTA head and neck planned for 3 months after discharge.

Our patient was diagnosed with the COL3A1 gene mutation, confirming the diagnosis of vEDS. Ongoing screening of vascular lesions was determined on an individual basis by vascular surgery. The interval and modality depends on the location and size of lesions. Genetics was also consulted to discuss the risk of inheritance and pregnancy with the patient and her family.

\section{DISCUSSION}

Diagnosis of vEDS is challenging because of variable penetrance within families. A family history of connective tissue disease can be absent as haploinsufficiency and biallelic sequence variants lead to variable clinical presentations and severity of illness ${ }^{7,8}$. De novo mutations in the COL3A1 gene are also a possibility as was suspected in our patient ${ }^{9}$.

There are 5 major and 12 minor criteria that distinguish vEDS from other forms of Ehlers-Danlos and help to establish a clinical diagnosis ${ }^{10}$. Of the major criteria-arterial vasculopathy at $<40$ years of age, spontaneous colon or uterine rupture, unexplained carotid-cavernous sinus fistulization, and family history - our patient had bowel perforation despite minimal trauma and early-onset vasculopathy. Several minor criteria were also present including easy bruising, thin skin (indicated by visible veins), characteristic facial features, joint hypermobility, and early-onset varicose veins ${ }^{10-12}$. Typical facial features include large eyes, small chin, lobeless ears, and thin nose and lips. However, characteristic facial features are often subtle and can be absent ${ }^{13}$. Joint hypermobility can be assessed by the Beighton score, a standardized evaluation for generalized joint hypermobility ${ }^{14}$. In patients with these features, vEDS should be suspected and genetic testing should be completed to confirm the diagnosis.

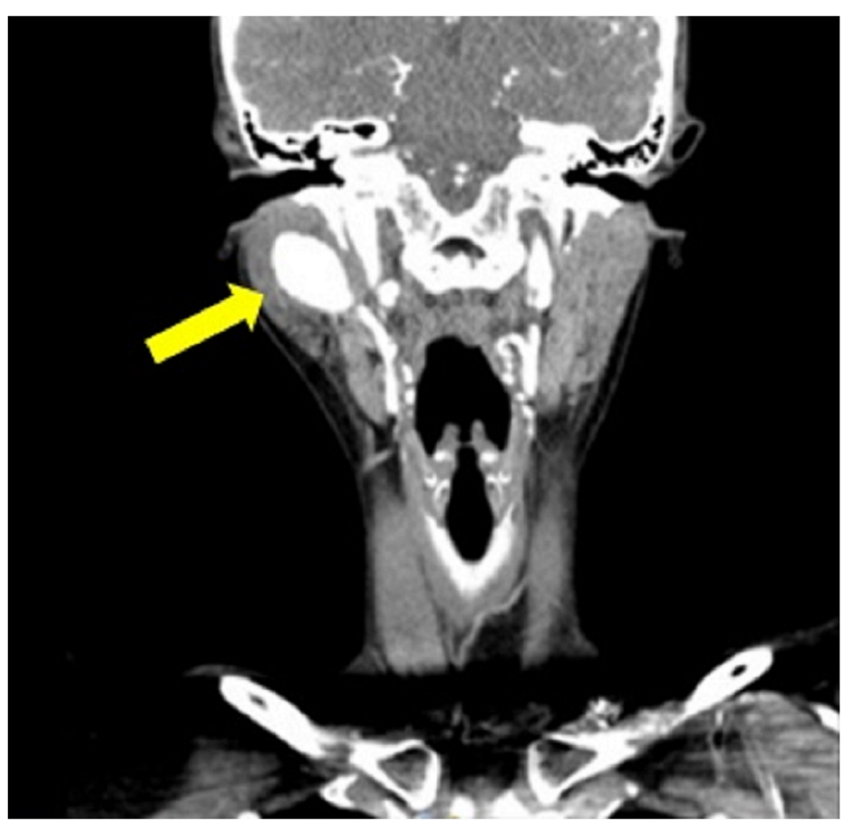

Figure 2. CTA demonstrating a large pseudoaneurysm of the maxillary artery. 
The diagnosis of vEDS is frequently delayed until after patients present with devastating sequelae. In one study, $61 \%$ of patients had at least one severe vascular complication prior to the age of 40 years ${ }^{15}$. Our patient was initially misdiagnosed with parotitis, but she was fortunate to return to care with a visible pulsatile mass that was treated prior to arterial rupture. However, most vascular abnormalities - including arterial aneurysms, dissections, and ectasias - are not visible on physical examination and occur in visceral arteries and deep arteries of the head and neck ${ }^{16}$. As such, practitioners should be aware that many patients first present with hemodynamic instability secondary to emergent internal bleeding. Although our patient was not bleeding, she was at high risk of rupture and urgent treatment of her aneurysm was necessary to prevent a catastrophic bleed.

The management of vascular complications in patients with vEDS is difficult. Endovascular procedures carry a significant risk of arterial dissection and aneurysm formation and are not typically recommended ${ }^{17}$. However, there are case reports of successful endovascular procedures in affected patients ${ }^{18,19}$. Our patient underwent open ligation of the external carotid artery rather than an endovascular approach in order to avoid arterial rupture and iatrogenic arterial damage. There is little evidence for effective medical management in vEDS. One randomized control trial demonstrated a significant reduction in arterial dissection and rupture with use of celiprolol, a $\beta 1$ $\beta 2$ receptor antagonist ${ }^{20}$. Our patient was discharged on carvedilol with the hope that other beta-blockers may offer a similar protective benefit. Oral contraception was warranted to help prevent pregnancy given the risk of uterine rupture. Combined oral contraception was preferred because progestin-only contraceptives have been associated with worsening joint hypermobility ${ }^{21}$.

Vascular Ehlers-Danlos has a relatively poor prognosis. The average life expectancy is 48 to 50 years. Patients typically die from acute arterial rupture leading to exsanguination ${ }^{22}$. Despite treatment, our patient has other aneurysms that pose a significant risk of bleeding and will require close outpatient follow-up. Following vascular lesions with non-invasive imaging has been shown to be an effective tool for counseling patients about the risks and benefits of elective surgical repair. However, patients should also be counseled that close monitoring does not change overall mortality, as fatal lesions are typically rapidly expansive ${ }^{23}$.

\section{CONCLUSION}

We describe a case of vEDS in a woman presenting with a pulsatile neck mass. This diagnosis requires a high degree of clinical suspicion and aggressive management to prevent catastrophic consequences. Clinicians should be aware that the vascular subtype can present with findings relatively dissimilar to the classic phenotype of Ehlers-Danlos. Endovascular approaches must be completed with caution and open ligation should be reserved for ruptured vessels or vessels in which rupture is imminent. Wider recognition of this unusual diagnosis will be essential to reducing the high morbidity and mortality associated with vEDS.

Corresponding Author: Bharat Maraj, MD; Department of Medicine University of California, San Francisco Medical Center, San Francisco, CA, USA (e-mail: bharat.maraj@ucsf.edu).

\section{Compliance with Ethical Standards:}

Conflict of interest: The authors declare that they do not have a conflict of interest.

\section{REFERENCES}

1. Germain DP, Herrera-Guzman Y. Vascular Ehlers-Danlos syndrome. Ann Genet 2004:47:1-9.

2. Barabas AP. Heterogeneity of the Ehlers-Danlos syndrome. Description of clinical types and a hypothesis to explain the basic defect. BMJ 1967;2:612-3.

3. Barabas AP. Vascular complications in the Ehlers-Danlos syndrome, with especial reference to the "arterial type" or Sack's syndrome. J Cardiovasc Surg 1972;13:160.

4. Pope FM, Martin GR, Lichtenstein JR, et al. Patients with EhlersDanlos syndrome type IV lack type III collagen. Proc Natl Acad Sci 1975;72(4):1314-6.

5. Oderich GS, Panneton JM, Bower TC, et al. The spectrum, management and clinical outcome of Ehlers-Danlos syndrome type IV: a 30-year experience. J Vasc Surg. 2005;42(1):98-106.

6. Kamiya K, Yoshizu A, Kashizaki F. Vascular-type Ehlers-Danlos syndrome incidentally diagnosed at surgical treatment for hemothorax; report of a case. Kyobu Geka. 2013;66(2):173-5.

7. Leistritz DF, Pepin MG, Schwarze U, et al. COL3Al haploinsufficiency results in a variety of Ehlers-Danlos syndrome type IV with delayed onset of complications and longer life expectancy. Genet Med. 2011;13(8):717-22.

8. Jørgensen A, Fagerheim T, Rand-Hendriksen S, et al. Vascular EhlersDanlos Syndrome in siblings with biallelic COL3A1 sequence variants and marked clinical variability in the extended family. Eur J Hum Genet. 2015;23(6):796-802.

9. Pepin M, Byers P. Ehlers-Danlos Syndrome, Vascular Type. Gene Reviews. Initial Posting: September 2, 1999; Last Update: May 3, 2011.

10. Malfait F, Francomano C, Byers P, et al. The 2017 international classification of the Ehlers-Danlos syndromes. Am J Med Genet Part C Semin Med Genet. 2017;175C:8-26.

11. Beighton P, De Paepe A, Steinmann B, et al. Ehlers-Danlos syndromes: revised nosology, Villefranche, 1997. Am J Med Genet 1998;77:31-7

12. Frank M, Says J, Denarié N, et al. Successful segmental thermal ablation of varicose saphenous veins in a patient with confirmed vascular Ehlers-Danlos syndrome. Phlebology. 2016;31(3):222-4.

13. Inokuchi $\mathbf{R}$, Kurata $\mathbf{H}$, Endo $\mathbf{K}$, et al. Vascular Ehlers-Danlos syndrome without the characteristic facial features: a case report. Medicine (Baltimore). 2014;93(28):e291.

14. Smits-Engelsman B, Klerks M, Kirby A. Beighton score: A valid measure for generalized hypermobility in children. J Pediatr. 2011;158:119-123123:e111-114.

15. Oderich GS, Panneton JM, Bower TC et al. The spectrum, management and clinical outcome of Ehlers-Danlos syndrome type IV: a 30-year experience. J Vasc Surg. 2005;42:98-106.

16. Zilocchi M, Macedo T, Oderich G, et al. Vascular Ehlers-Danlos Syndrome: Imaging Findings. Am J Rad. 2007;189:712-719.

17. Bergqvist D, Björck M, Wanhainen A. Treatment of vascular EhlersDanlos syndrome: a systematic review. Annals of Surgery. 2013;258(2):257-261.

18. Linfante I, Lin E, Knott E, et al. Endovascular repair of direct carotidcavernous fistula in Ehlers-Danlos type IV. J Neurointerv Surg. 2015;7(1):e3.

19. Iida Y, Obitsu Y, Komai H, et al. Successful coil embolization for rupture of the subclavian artery associated with Ehlers-Danlos syndrome type IV. J Vasc Surg. 2009;50(5):1191-5. 
20. Ong KT, Perdu J, De Backer J, et al. Effect of celiprolol on prevention of cardiovascular events in vascular Ehlers-Danlos syndrome: a prospective randomised, open, blinded-endpoints trial. Lancet. 2010;376(9751):1476-84.

21. Hugon-Rodin J, Lebegue G, Becourt S, et al. Gynecologic symptoms and the influence on reproductive life in 386 women with hypermobility type ehlers-danlos syndrome: a cohort study. Orphanet J Rare Dis. 2016;11(1): 124 .
22. Shields LBE, Rolf CM, Davis GJ, et al. Sudden and unexpected death in three cases of Ehlers-Danlos syndrome type IV. J Forensic Sciences. 2010;55(6): 1641-1645.

23. Callewaert B, Malfait F, Loeys B, et al. Ehlers-Danlos syndrome and Marfan syndrome; Best Practice and Research. Clinical Rheumatology. 2008;22:165-189. 